\title{
Experimental study of pressure drop reduction on in-line tube bundle using passive control
}

\author{
Omar Ladjedel ${ }^{1, a}$, Lahouari Adjlout ${ }^{1},{\text { Tayeb } \text { Yahiaoui }^{2} \text { and Omar Imine }}^{2}$ \\ 1 Laboratoire d'aérohydrodynamique navale, Département de génie maritime, Mechanical Engineering Faculty, \\ 31000 USTO Oran, Algeria \\ 2 Laboratoire d'aéronautique et systèmes propulsifs, Département de Génie Mécanique, Mechanical Engineering Faculty, \\ 31000 USTO Oran, Algeria
}

Received 3 July 2012, Accepted 30 June 2013

\begin{abstract}
In the present paper an experimental investigation of drag reduction on in-line tube bundles with a configuration of seven by seven with a pitch ratio of 1.44 has been performed. Two longitudinal grooves are placed on the external surface of the circular cylinders as a passive control. The experiment is carried out using a subsonic wind tunnel. The pressure distributions for various azimuthal angles along the tubes are measured using a multi-channel differential pressure. Drag forces are also determined using a combined wire-strain gauge balance. The pressure drops are deduced from the resulting drag forces. The results obtained show an unexpected reduction in the pressure drop for the tube bundles. A reduction of $36.5 \%$ in the pressure drop for a Reynolds number of $1.33 \times 10^{4}$ is found compared to the configuration without grooves. Some correlations for the pressure drop are proposed.
\end{abstract}

Key words: Circular cylinder / tube bundles / pressure distribution / drag / grooves / Reynolds number

\section{Introduction}

The turbulence provided by coherent vorticity phenomenon may lead to severe vibrations thus causing extensive damage to tube bundles over a short period of time. Hence there are many issues that need to be addressed and resolved properly. One such concern is the strong fluid-structure coupling which acts as a cyclic load on tubes inside the heat exchangers.

Most previous experimental studies on the turbulent flow over tube bundles have focused on measuring the heat transfer and pressure drop. For example, Pierson [1] studied heat transfer and pressure drop across the tube bundle arrangements in various configurations in the cross flow of gases. Zukauskas and Ulinskas [2] presented some experimental results on both the characteristics of flow and heat transfer tubes and tube bundles in cross flow. They found that the pressure drop across a tube bundle decreases with the Reynolds number. They also reported correlations for pressure drop and heat transfer coefficients.

The features of the flow change when using two cylinders compared with the flow pattern for isolated cylinder. Arie et al. [3] showed that for a tandem arrangement

a Corresponding author: ladjedelomar@yahoo.com

\section{Nomenclature}

\begin{tabular}{|lll|}
\hline$C_{\mathrm{P}}$ & Pressure coefficient & $(-)$ \\
$C_{\mathrm{D}}, C_{\mathrm{L}}$ & Drag and lift coefficient & $(-)$ \\
$p$ & Pressure & $\left(\mathrm{N} . \mathrm{m}^{-2}\right)$ \\
$d$ & Cylinder diameter & $(\mathrm{m})$ \\
$L$ & Length of the cylinder & $(\mathrm{m})$ \\
$S$ & Projected area of the cylinder & $\left(\mathrm{m}^{2}\right)$ \\
$U_{0}$ & Inlet velocity & $\left(\mathrm{m} . \mathrm{s}^{-1}\right)$ \\
$U_{\mathrm{g}}$ & Gap velocity & $\left(\mathrm{m} . \mathrm{s}^{-1}\right)$ \\
$R e$ & Reynolds number & $(-)$ \\
$\Delta P$ & Pressure drop & $\left(\mathrm{N} \cdot \mathrm{m}^{-2}\right)$ \\
\hline \multicolumn{3}{|c}{ Greek letters } \\
\hline$\rho$ & Fluid density & $\left(\mathrm{Kg} \cdot \mathrm{m}^{-3}\right)$ \\
\hline
\end{tabular}

the fluctuating lift and drag on the downstream cylinder is strongly dependent upon the gap spacing. It is shown that as the gap spacing increases, the fluctuating forces also increase. It is concluded by the authors that the worst case scenario is at a gap distance of $4.5 \mathrm{D}$, where the fluctuating forces on the downstream cylinder are the maximum. The papers of Chen [4] and Pettigrew et al. [5] contain extensive data for lift and drag forces for various tube bundle configurations; in-line, triangular and staggered arrangements. There are some other interesting configurations such that of $\mathrm{Wu}$ et al. [6] in which a 
cylinder is placed behind another one but with a little offset from the centre line. The upstream cylinder is always fixed whereas the downstream cylinder is tested at various positions to see which configuration results in small lift and drag forces. The idea behind this study is to find an optimal location for the downstream cylinder to provide the needs of risers in marine engineering. It is concluded that the downstream cylinder will always have a non-zero lift and drag.

When two or more cylinders are placed close to each other the interference between them can be of three types. Proximity interference, this type of interference is observed for cases where the cylinders are placed very close to each other especially in tandem or in-line arrangements. Wake interference is observed when the cylinders are placed widely apart from each other. In such a case the first cylinder behaves as a single cylinder whereas the downstream cylinder flow behavior is dependent upon the wake of the upstream cylinder. The third case is of mixed effect of proximity and wake, in which the cylinders are placed at a moderate distance from each other, Afgan [7].

According to Mittal and Kumar [8] the behavior of flow past two stationary cylinders is still not very clear and it is impossible to predict the response of oscillating cylinders. Wolfe and Zaida [9] used two tandem cylinders to study a feedback control on vortex shedding. It is concluded that when a cylinder is placed in the wake of another cylinder then its unsteady loading is not only dependent upon the flow behavior in its own wake but also on the flow pattern in the wake of the upstream cylinder. Lam and Lo [10] have conducted a visualization study for $P / D$ ranging from 1.28 to $5.96, L / D=21.3$ and $R e=2.1 \times 10^{3}$. The flow characteristics are described by three flow patterns: (i) the downstream cylinder is shielded by the shear layers of the upstream cylinder, (ii) the free shear layers from the upstream cylinder are reattached onto the downstream cylinder and (iii) the downstream cylinder is impinged by the vortices shed from the upstream cylinder.

Some experimental studies on the flow around four cylinders in an in-line square configuration have been the focus of many researchers. Sayers [11] has measured the lift and drag coefficients on a single cylinder in a group of four equally spaced cylinders in an open-jet wind tunnel for $P / D$ ranging from 1.1 to 5.0 and $R e=3.0 \times 10^{4}$. A comparison with flow interference of a group of three cylinders shows that there exists similarly obtained data to form a basic code of design. Later, Sayers [12] further determined the Strouhal number of each cylinder in the same open-jet wind tunnel. The study indicated that at $P / D \geq 4.0$, St is equal to those for flow around a single isolated cylinder, while at $P / D<4.0$, St varies across the wake. Lam and Fang [13] have studied experimentally the effects of flow interference of four-cylinder array on the mean pressure distribution and force coefficients of the cylinders, various $P / D$ are investigated for $R e=12.8 \times 10^{3}$. Lam et al. [14] measured the mean and fluctuating lift and drag coefficients on the four cylinders at $P / D$ from 1.69 to 3.83 and $R e=41.0 \times 10^{3}$ giving additional insight on the flow-induced forces. Further experimental investigations (Lam et al. [15-17]) using a DPIV (digital particle imaging velocimetry) method and an LIF (laser induced fluorescence) flow visualization technique confirmed the characteristics of the flow pattern observed around the four cylinders in an in-line square configuration. Based on these studies, it is possible to understand the flow physics and the relationship between flow pattern transformation and $P / D$, the mean and fluctuating force and pressure characteristics on the cylinder. Furthermore, these experimental results were limited to fairly high $R e$; little is known of the phenomena at low $R e$. One of the reasons could be due to measurement difficulties at low $R e$. Another reason is that most engineering applications occur at high $R e$. Contrary to experimental studies, numerical simulation has obvious advantages in low-Re flow investigations. It can quickly provide flow information, such as the instantaneous full-field information of the velocity field, the vorticity field and the pressure field, which are very difficult to obtain experimentally.

Pressure drop across the tube bank has been investigated by Grimison [18] and Zukauskas [19]. Results have shown that increasing Reynolds number and decreasing transverse tube spacing cause an increase in the pressure drop. The longitudinal spacing is found to have no effect on the tube array pressure drop. The largest pressure drop is observed across a bank with closely spaced tubes. Yahiaoui et al. [20] have performed an experimental study about the turbulent flow around an in-line tube bundle with a $P / D=1.44$ for a Reynolds number $R e=27400$ (based on the speed between the tubes). They found that the flow through the tube bundle is asymmetrical, the stagnation point is located at 45 degrees and that the drag forces are higher in the first row.

A comparison of drag coefficients for various Reynolds numbers (based on diameter and free stream velocity) and an aspect ratio $L / D=11.5$ between smooth cylinder and the cylinder fitted with two longitudinal grooves is investigated experimentally by Ladjedel et al. [21]. They found that for a Reynolds number $R e=8.6 \times 10^{4}$ the cylinder fitted with two grooves reduces the drag force about $17.51 \%$.

The purpose of the present study is to investigate experimentally the pressure drop reduction on in-line tube bundle using flow control by adding grooves to the tubes and compare the obtained result with that of Yahiaoui [22].

\section{Apparatus and experimental procedure}

\subsection{Wind tunnel and the bundle rig}

The experiments are carried out in closed circuit wind tunnel. The test section is of square cross-section $0.46 \mathrm{~m} \times$ $0.46 \mathrm{~m}$ and $1.2 \mathrm{~m}$ long. The closed circuit wind tunnel is of conventional design in which the velocity could reach $60 \mathrm{~m} . \mathrm{s}^{-1}$ and the turbulence intensity is less than $1 \%$.

The test section is composed of seven rows of rigid PVC tubes of an identical external diameter of $40 \mathrm{~mm}$ 


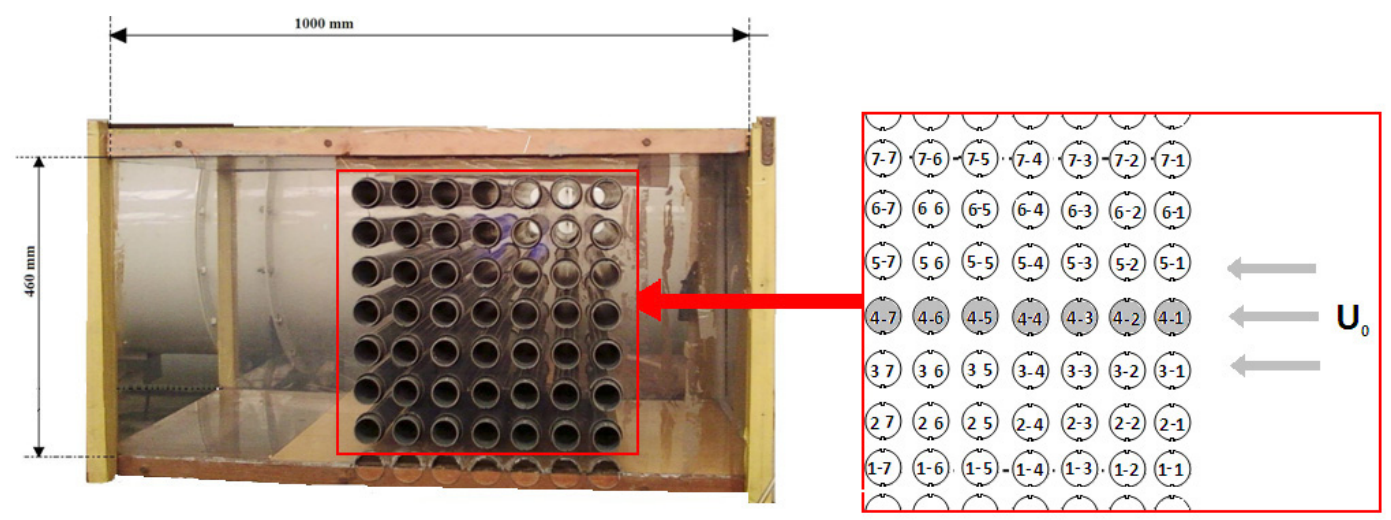

Fig. 1. Working section and geometrical features of the tube bundle.

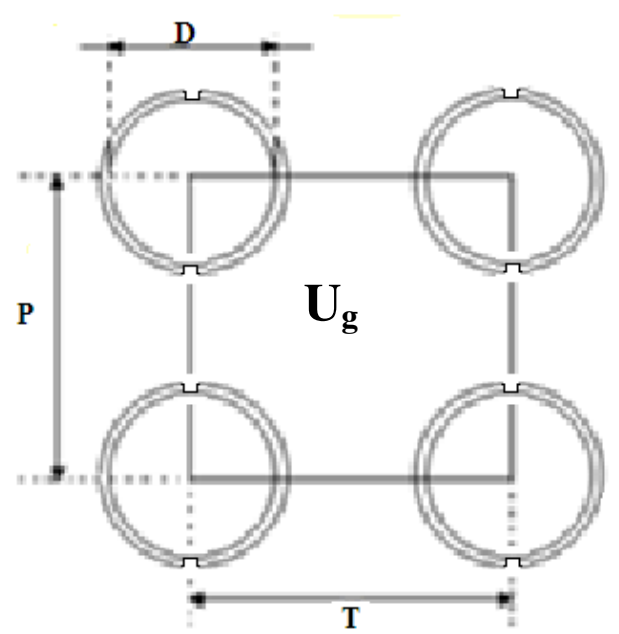

Fig. 2. Pitch ratios of tube bundles.

and $460 \mathrm{~mm}$ length. Cylinders are in in-line equispaced arrangement. As shown in Figure 1, each row has seven full tubes. Half tubes were also mounted along the bottom and top walls of the test model alternately to simulate an infinite tube bundle and minimize the wall boundary layer. The spacing ratio is defined as the centre-to-centre distance of adjacent cylinders divided by the cylinder diameter. A schematic diagram of the arrangement is shown in Figure 2.

The experiment setup is shown in Figure 3. One Pilot cylinder is equipped with 22 pressure tapings on its circumference at mid-span and two other planes at the both ends. The diameter of the tapings is $0.5 \mathrm{~mm}$. The shape and dimensions of the groove are shown in Figure 3c. The pressure measurements are carried out for the 49 tubes for an azimuthal angle from $0^{\circ}$ to $360^{\circ}$. Drag forces for all the tubes and the tube bundle are determined using the TE81 balance and the pressure distributions on the cylinder are measured using a TE44DPS differential pressure with a multi-channel scanner. The latter pressure scanning box allows sequential selection of up to 20 pressure tapings. The display unit links to a computer, loaded with DATASLIM software for data analysis.
To measure the total drag force of the tube bundle, an implemented device is designed and is shown in Figure 4. The testing rig is installed on a carriage which assures the free translation of the tube bundle with a minimal friction force. The junction between the input of the test rig and the exit of the wind tunnel convergent is ensured by using a flexible membrane; initially this junction allows the passage of the air from the wind tunnel to the tube bundle as well as the free movement of the testing rig. The balance is fixed on the table which supports the carriage. The stem of the balance is fixed in the tube bundle.

The pressure difference between the inlet and the outlet of the tube bundle creates the movement of the carriage, from where the force of the translation is transmitted directly to the balance.

\section{Result and discussion}

The experiments are performed for the following parameters:

- pitch ratio, $P / D=1.44(P=T=57.6 \mathrm{~mm}, D=$ $40 \mathrm{~mm})$;

- Free stream and gap bulk velocity: $U_{0}=3.1321 \mathrm{~m} . \mathrm{s}^{-1}$, $U_{\mathrm{g}}=10.2505 \mathrm{~m} . \mathrm{s}^{-1}\left(U_{0}\right.$ mean flow velocity, $U_{\mathrm{g}}$ gap bulk velocity);

- Reynolds number $=27400$ (based on diameter and gap bulk velocity).

\subsection{Pressure, drag and lift coefficients}

The coefficient of pressure is defined as:

$$
\begin{aligned}
C_{\mathrm{P}} & =\frac{p-p_{\mathrm{ref}}}{0.5 \times\left(\rho U_{\mathrm{g}}^{2}\right)} \\
U_{\mathrm{g}} & =\frac{U_{0}}{\left(1-\frac{D}{T}\right)}
\end{aligned}
$$

Measurement starts from the frontal point facing the mainstream flow and ends at the same point after a complete revolution. The average value is then calculated when the Pilot cylinder changes position in the matrix. 


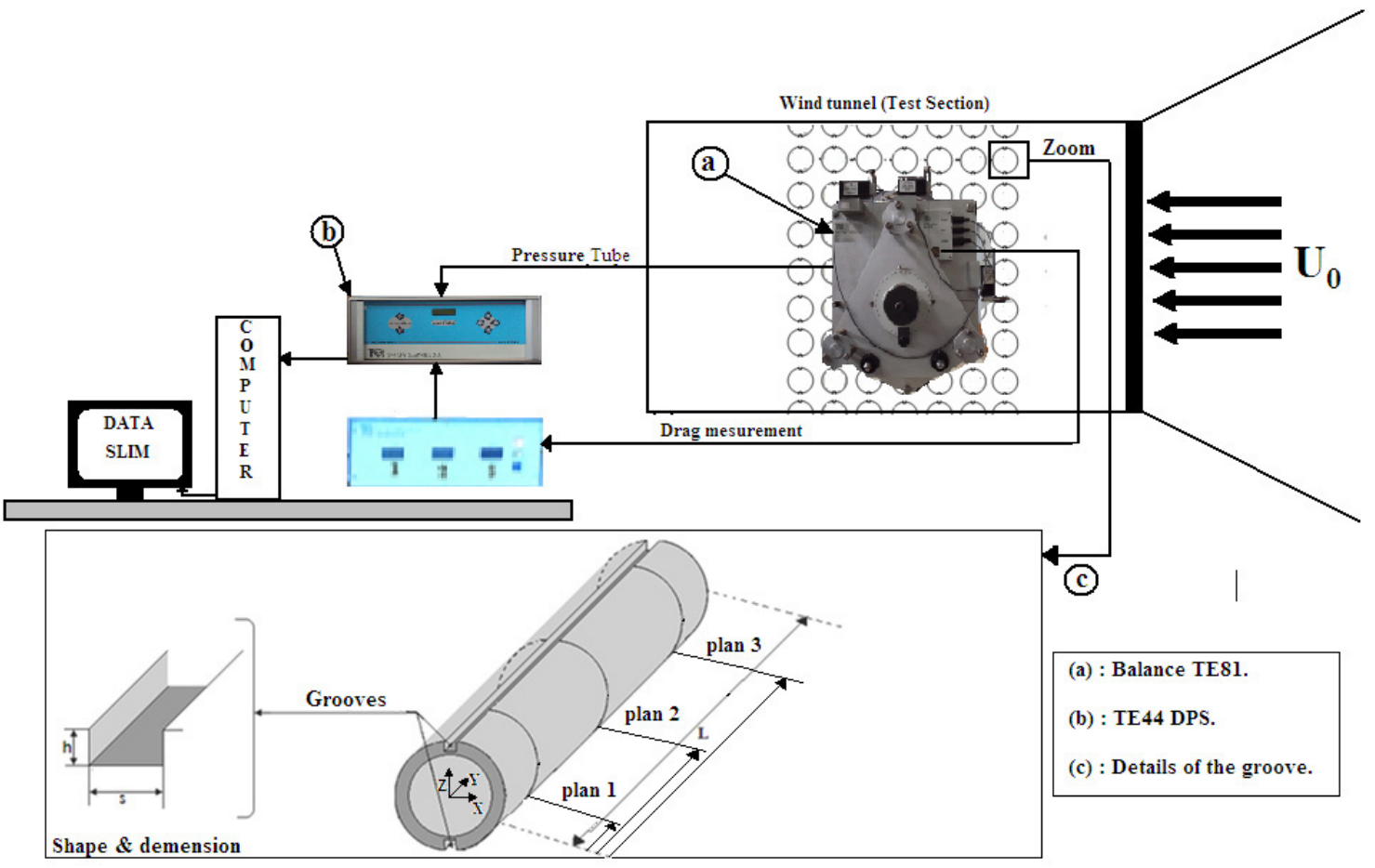

Fig. 3. Experimental set-up.

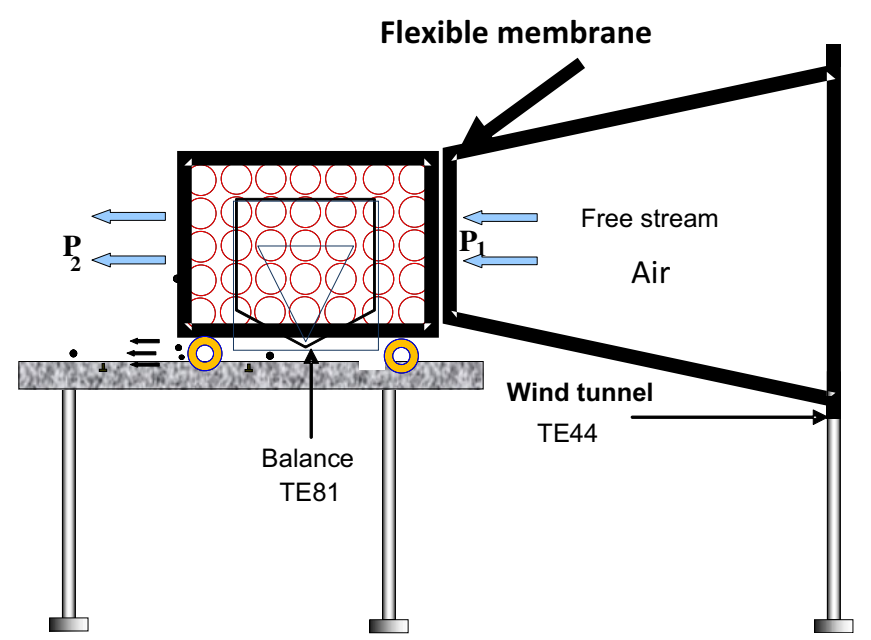

Fig. 4. Tube bundle drag measurement set-up.

In Figure 5, mean pressure distributions around the different tubes for the 49 positions at the mid-span are presented. The pressure distribution on cylinder $4-1$ is almost a symmetry mapping resulting in a low lift force. In the pressure recovery region behind the cylinders the profile becomes flat. Similar behavior is also found by Lam and Fang [13]. The same trend is observed for the other cylinders of the first row. However for the remaining rows, unsymmetrical distributions are obtained. In the next section, only the distributions for the cylinder four for all the rows are discussed.

Figure 5a shows the pressure distributions for the tubes on the first row. The obtained distributions seem to be similar to that of an isolated cylinder. It is noticed that the stagnation point is located at an angle of $0^{\circ}$ degree which corresponds to the maximum pressure. The interaction of the other rows on the first row appears to be negligible.

The maximum of the pressure for the second row shifts from the frontal point to the inner side about $\left(\theta=315^{\circ}\right)$. This is due to the reattachment of the shear layer from the upstream tube. From this point, the pressure decreases leading to flow acceleration. Then, an increase in the pressure gradient appears causing the separation of the reattached shear layer. The latter creates a narrow wake.

However, for the remaining rows the stagnation point is almost located at the same position $\left(\theta=40^{\circ}\right)$. In the range $90^{\circ}-100^{\circ}$ the pressure coefficient has a minimum value; this interval represents the region of shear layer separation. It has to be noticed that the pressure coefficient distribution for row seven tends to be symmetrical. The non-symmetrical pressure distributions for rows two to seven lead to a non-zero lift force. This is probably due to the deviation of the jet flow between the tubes of the previous rows. This phenomenon has been discussed by many authors Le Gal et al. [23], Ishigai et al. [24] and Auger et al. [25]. When the distance between the cylinders is less than two diameters as is for the present study, the Coanda effect seems to be the cause of the jet deviation between tubes, Masanori et al. [26]. A very different behavior for the pressure distribution is observed for tubes $1-5$ and $7-5$. This is probably due to the fact that these cylinders are positioned near the wall.

In the wake region, a pressure measurement scattering is observed for the first row due probably to high 


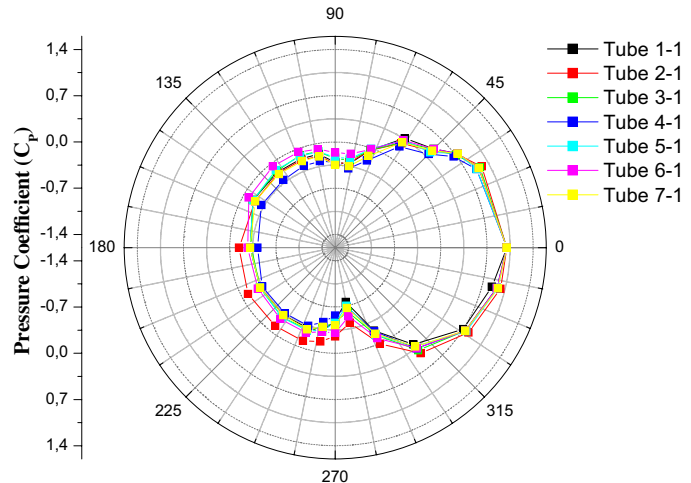

(a) Row 1

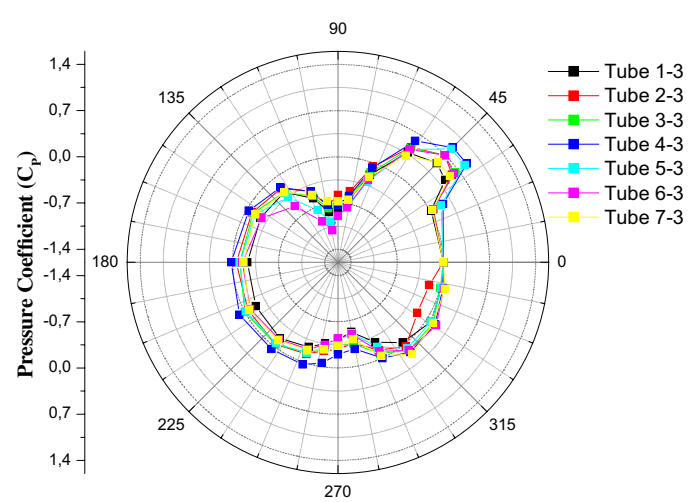

(c) Row 3

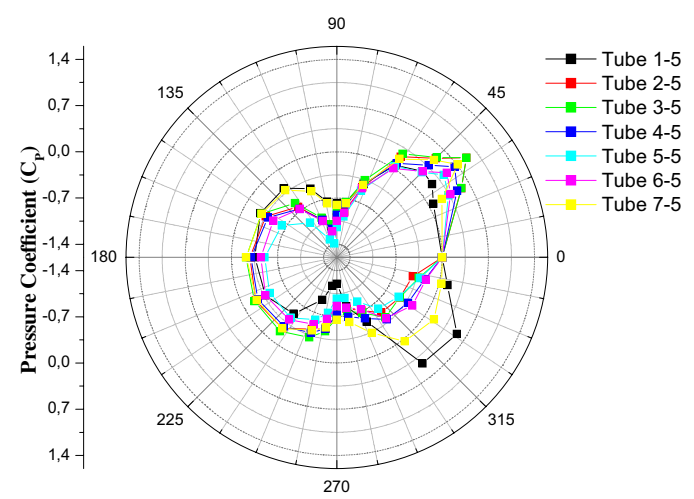

(e) Row 5

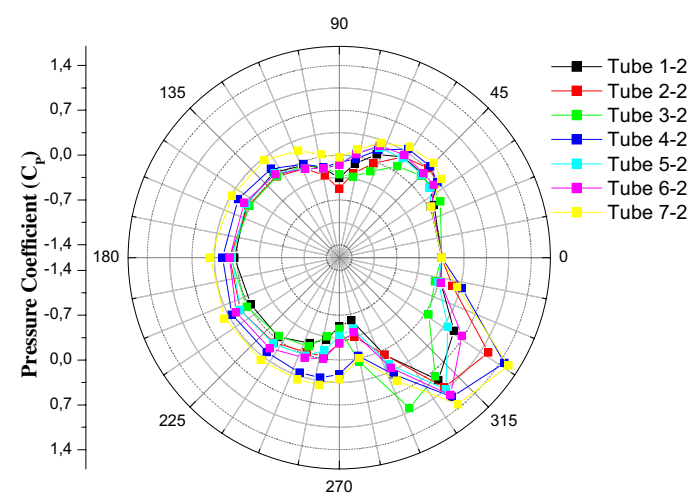

(b) Row 2

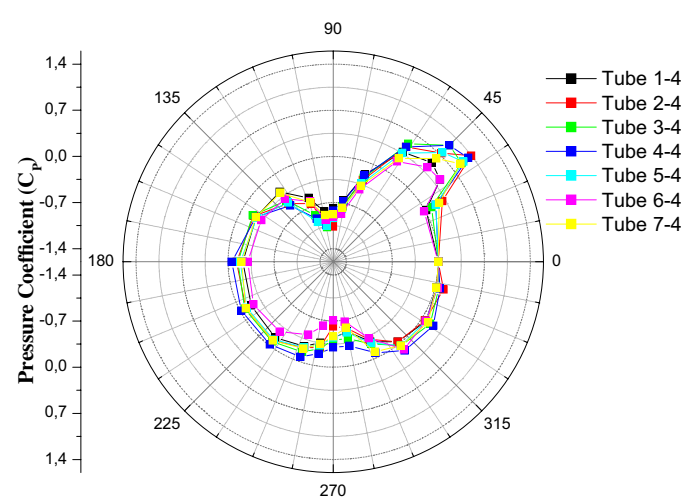

(d) Row 4

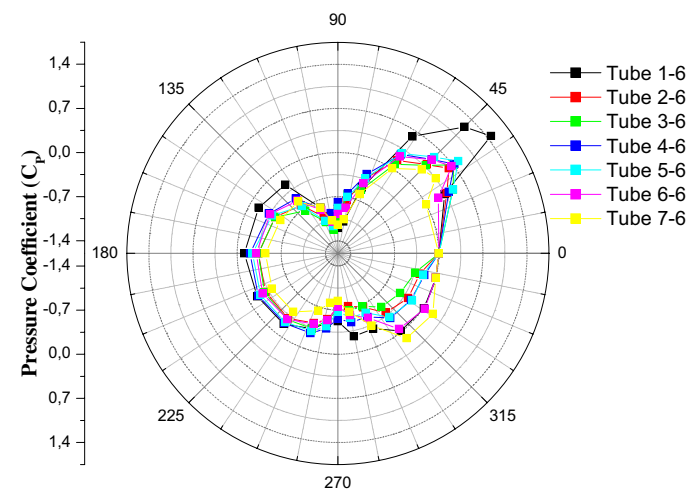

(f) Row 6

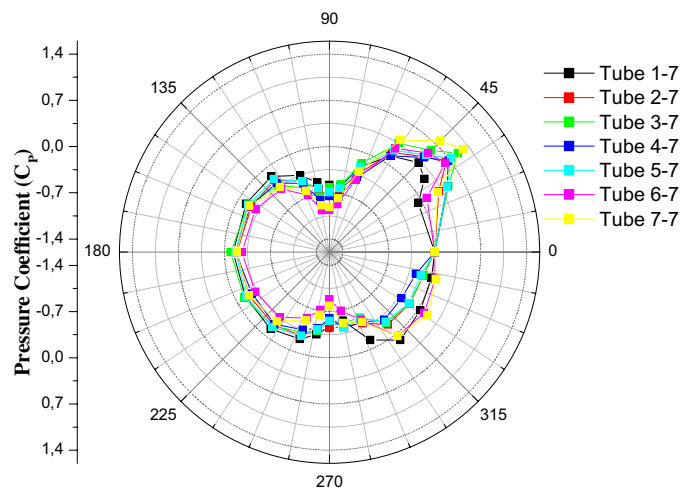

(g) Row 7

Fig. 5. Mean pressure coefficient distribution for different tubes. 
Table 1. Different drag coefficients for line 4.

\begin{tabular}{cccc}
\hline Tube & CD Balance & CD Integral & CD Interference Balance \\
\hline $4-1$ & 0.96567 & 0.95302 & 0.14833 \\
$4-2$ & 0.217 & 0.23784 & 0.923 \\
$4-3$ & 0.42223 & 0.2116 & 0.69177 \\
$4-4$ & 0.34368 & 0.37122 & 0.77032 \\
$4-5$ & 0.22358 & 0.34352 & 0.89042 \\
$4-6$ & 0.51622 & 0.30712 & 0.59778 \\
$4-7$ & 0.38057 & 0.22613 & 0.73343 \\
\hline
\end{tabular}

turbulence intensity of the flow. This remark is valid for the remaining rows.

In the present case, for the Reynolds number investigated the drag pressure is dominant relative to the friction drag. Therefore the pressure distribution is enough to determine of the drag and lift forces. For the first row, a high drag force is noted.

By integrating the pressure distributions over the surface of each cylinder, the drag and lift coefficients are given as follows:

$$
\begin{aligned}
& C_{\mathrm{D}}=\frac{1}{2} \int_{0}^{2 \pi} C_{\mathrm{p}}(\theta) \cos (\theta) \mathrm{d} \theta \\
& C_{\mathrm{L}}=-\frac{1}{2} \int_{0}^{2 \pi} C_{\mathrm{p}}(\theta) \sin (\theta) \mathrm{d} \theta
\end{aligned}
$$

Figure 6 shows the distributions of the drag and lift coefficients for the 49 tubes for different planes where the Planes 1,2 and 3 are respectively situated at $L / D=$ $2.875 ; 5.75$ and 8.625 for $R e=27400$. It is clearly seen that there is a non-homogeneous distribution of forces on the surface along the tubes. A high fluctuation of pressure in the center of the tube is observed compared with those measured at the two ends. The forces at the ends of the tubes are almost similar.

Table 1 shows only the drag coefficients obtained by the balance and by the pressure coefficients integration at the mid- span of the tubes for line 4 . It has to be noted that the balance measures the drag force for the whole cylinder; however the pressure coefficient integration is performed at plane 2. The differences between the two obtained $C_{\mathrm{D}}$ is due to a non-uniform drag distribution along the tube.

The values of the $C_{\text {Dinterference }}$ are also presented in the above table. The interference drag coefficient defined by Zdravkovich [27] as the difference between the drag coefficient measured on the cylinder in the configuration and the drag coefficient of a single cylinder at the same Reynolds number is adopted in the present study. The drag coefficient for a grooved single cylinder is taken from reference [18], $C_{\mathrm{D}}=1.14$.

The variations of the drag interference coefficients reveal how the interaction of cylinders is affected by the position of each cylinder in the tube bundle as confirmed by Figure 7 which shows the drag coefficient distributions for all tubes obtained by balance and by pressure integration.

Figures 8 show the drag coefficient evolutions for each cylinder with a variation of Reynolds number.
The curves show nearly the same trend for all the cylinder positions in the tube bundle.

For a given Reynolds number, very high values of the drag force are observed for the first row of tube bundle. While for the second row the drag force decreases sharply where it takes a minimum value for all positions of the cylinder due to the wake area of the first row. The drag force then seems to stabilize for the remaining rows.

\subsection{Pressure drop}

The driving power in hydraulic system is directly related to the pressure drop. Using the momentum theorem, the pressure drop through the in line tube bundle is calculated as follows:

$$
\Delta p=\frac{D_{T}}{A}
$$

where: $D_{\mathrm{T}}$ is total drag $[\mathrm{N}]$ and $\mathrm{A}$ is the area of the inlet section $\left[\mathrm{m}^{2}\right]$.

Several Reynolds numbers are investigated to determine the pressure drop evolution and a comparison with the results obtained by Yahiaoui [22] is made. In the latter work, high values for drag force were obtained in row 1 and 5. In the present investigation, two tests were performed. The first case consists in replacing the cylinders of row 1 and 5 by grooved tubes while the second case concerns the tube bundle with all grooved cylinders.

Figure 9 shows a comparison of the pressure drop variation in tube bundle with smooth cylinders and the present study. It is clearly seen an increase in the pressure drop with an increase in the Reynolds number. The pressure drops for both cases are lower compared with the curve of reference [22]. The tendencies for both cases seem to be quadratic. However the curve obtained by reference [22] for smooth cylinder appears to be linear. Mehrabian [28] confirms a linear trend between the velocity head upstream of the tube bank and the pressure drop.

Table 2 shows the pressure drop reduction for various Reynolds numbers compared with smooth cylinder tube bundle. Significant reductions in pressure drop are noticed. The table also shows that the reduction rate decreases with increasing Reynolds number for both tests. It should be noted that the use of the grooved cylinder across the tube bundle (case 2 ) shows highest reduction in pressure drop. A reduction of $36.5 \%$ in the pressure drop for a Reynolds number of $1.33 \times 10^{4}$ is found compared to the configuration without grooves. 


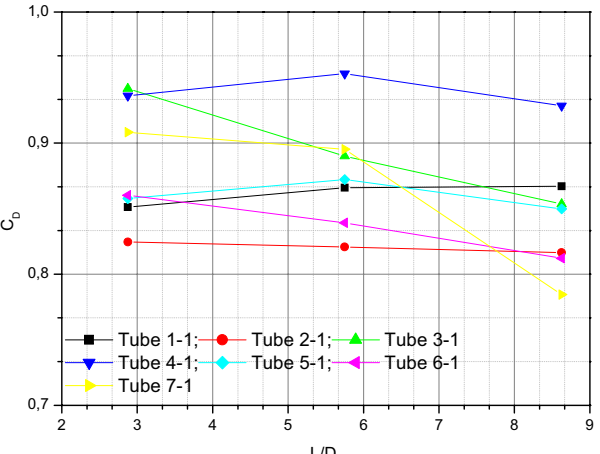

Drag coefficient

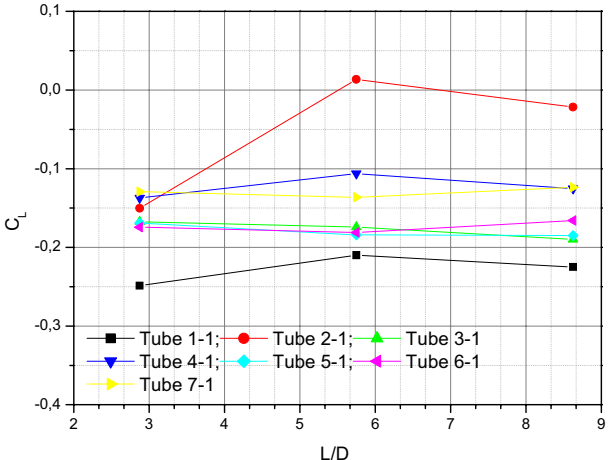

Lift coefficient

(a) Row 1

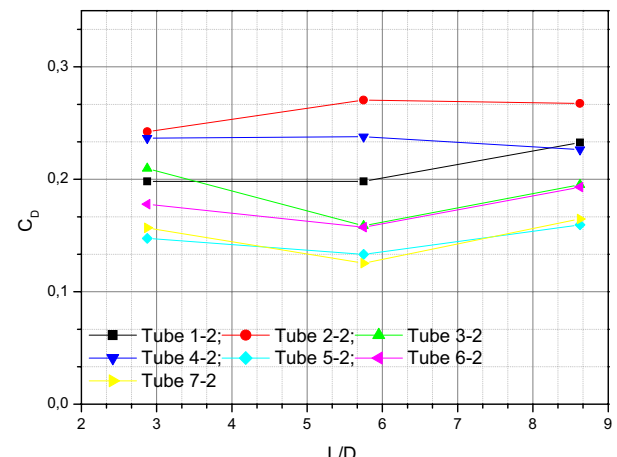

Drag coefficient

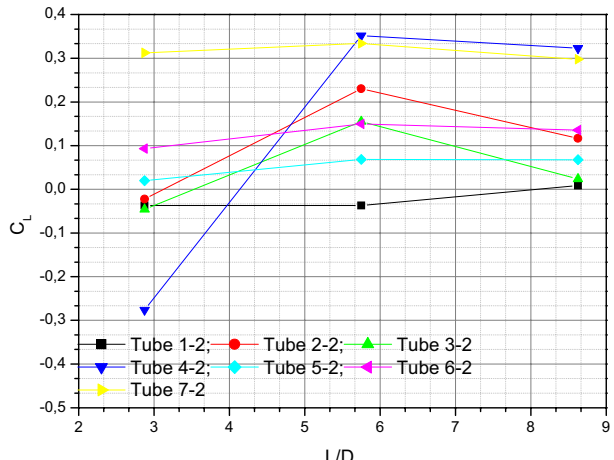

Lift coefficient

(b) Row 2

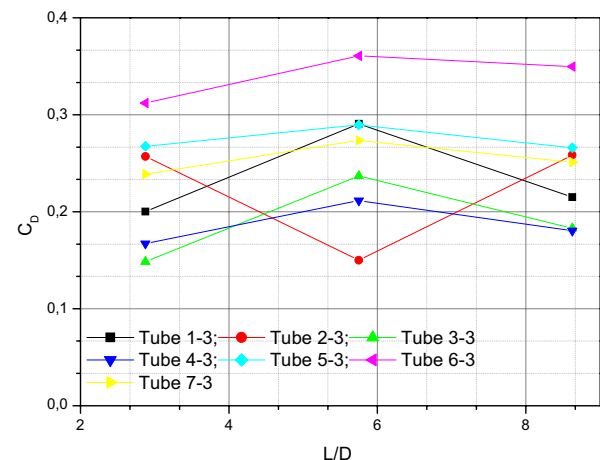

Drag coefficient

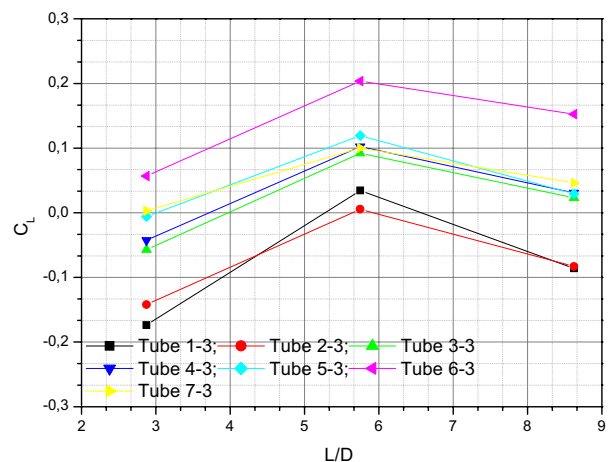

Lift coefficient

(c) Row 3



Drag coefficient

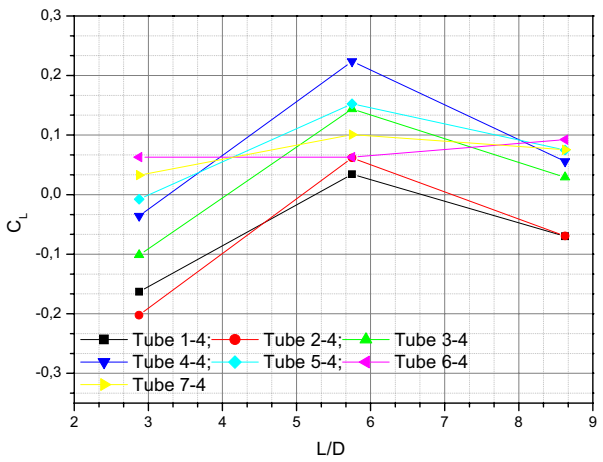

Lift coefficient

(d) Row 4

Fig. 6. Drag and lift coefficients distributions along the tube length for $R e=27400$. 


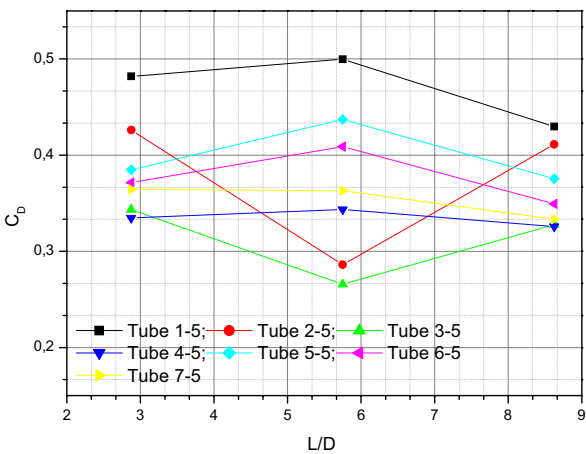

Drag coefficient

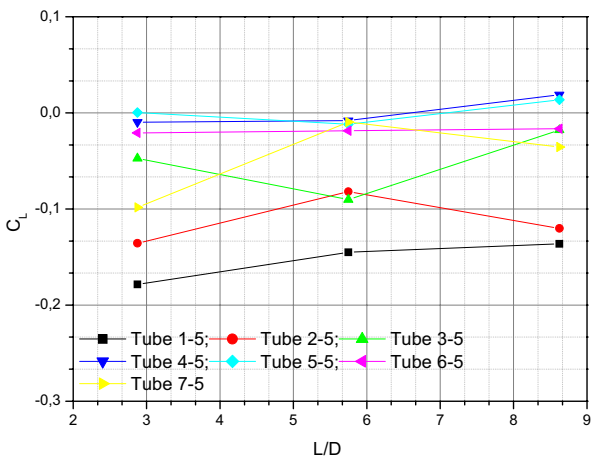

Lift coefficient

(e) Row 5

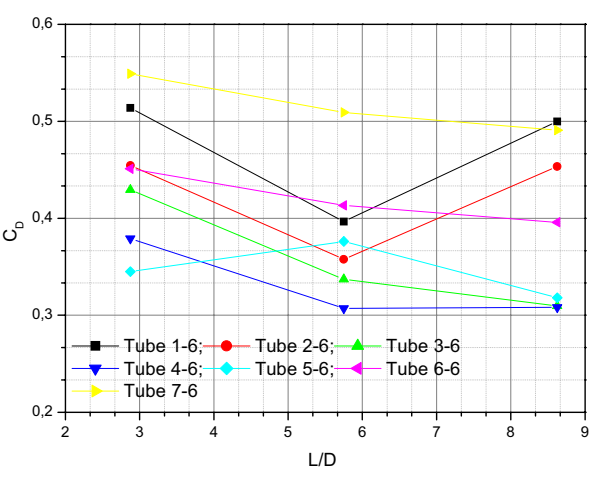

Drag coefficient

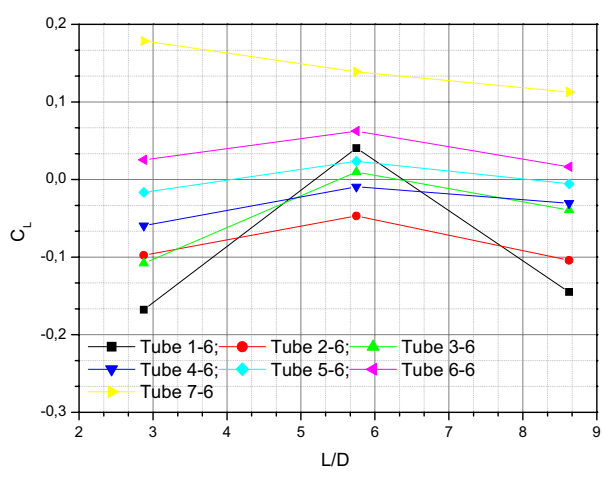

Lift coefficient

(f) Row 6



Drag coefficient

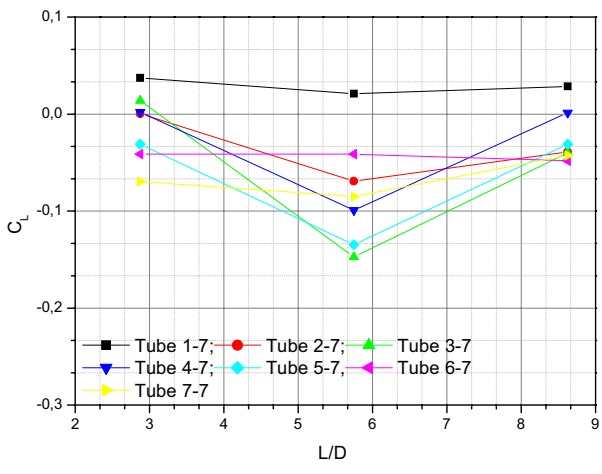

Lift coefficient

(g) Row 7

Fig. 6. Continued.

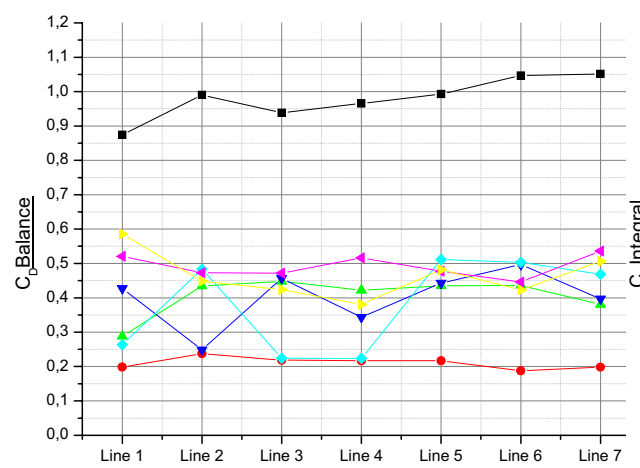

(a)

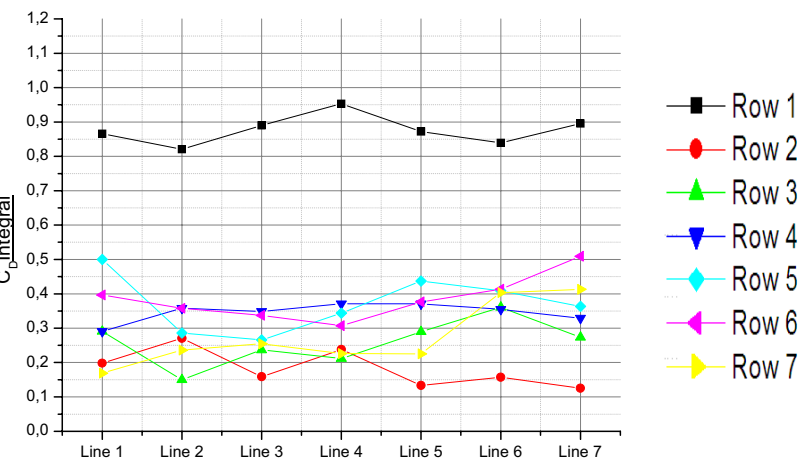

(b)

Fig. 7. Drag coefficient distributions for all tubes, (a) balance (b) pressure integration. 


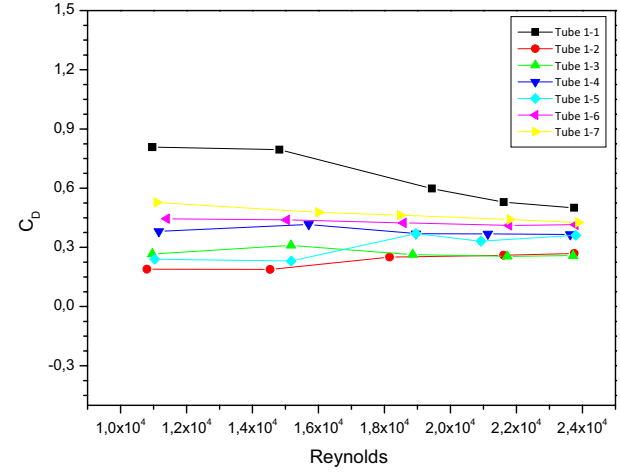

(a) Row 1

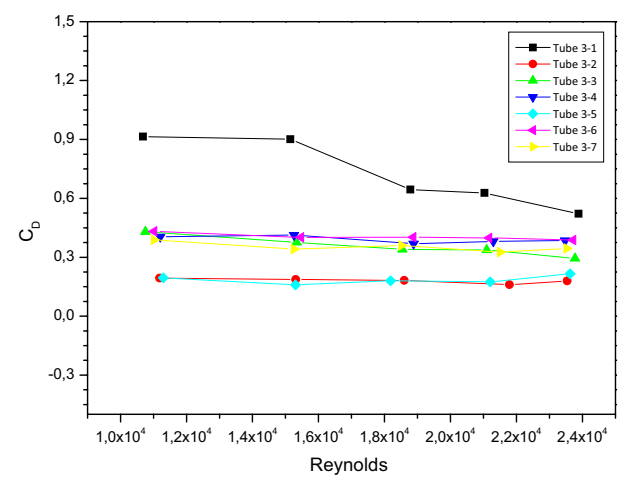

(c) Row 3

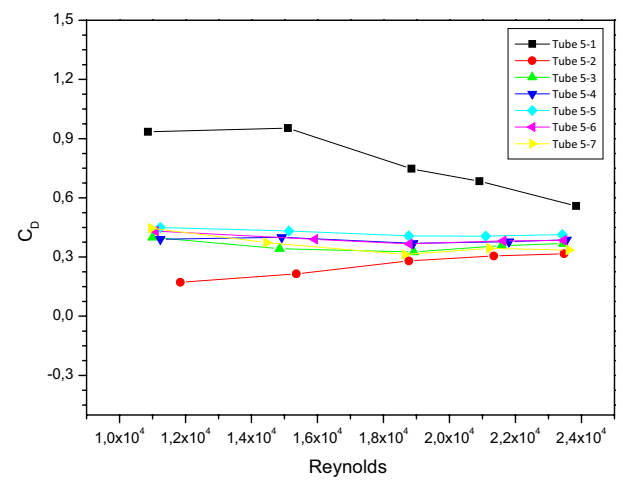

(e) Row 5

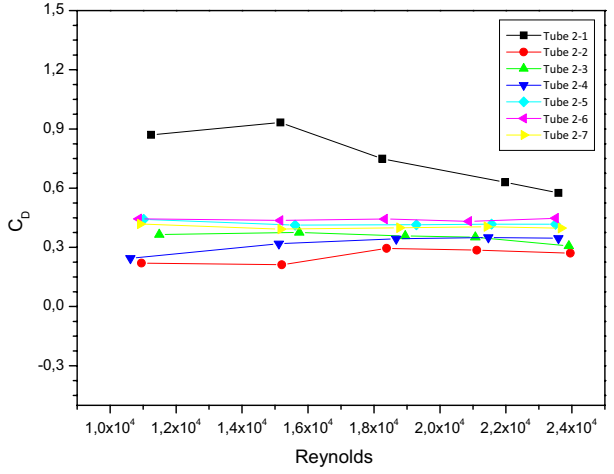

(b) Row 2

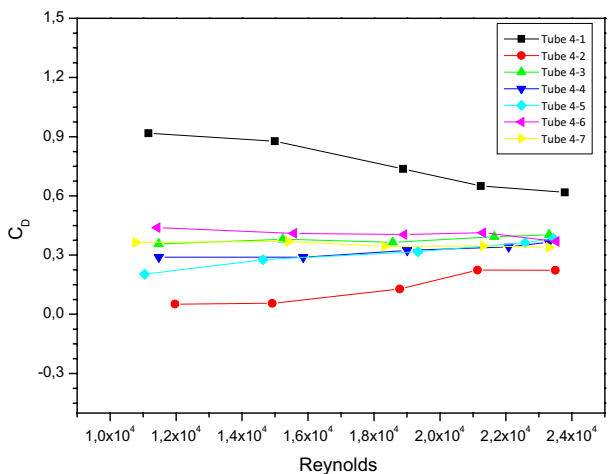

(d) Row 4

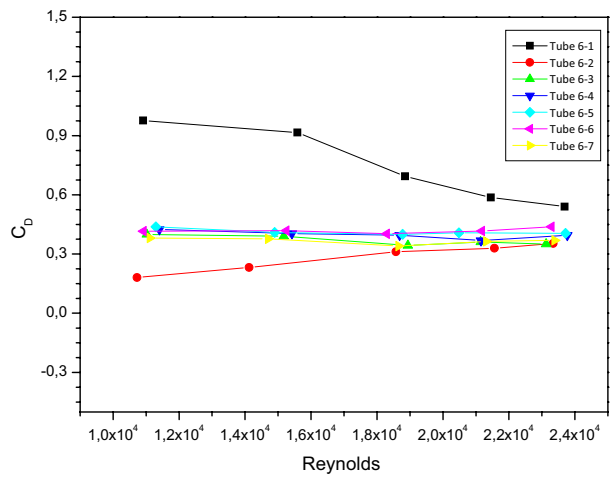

(f) Row 6

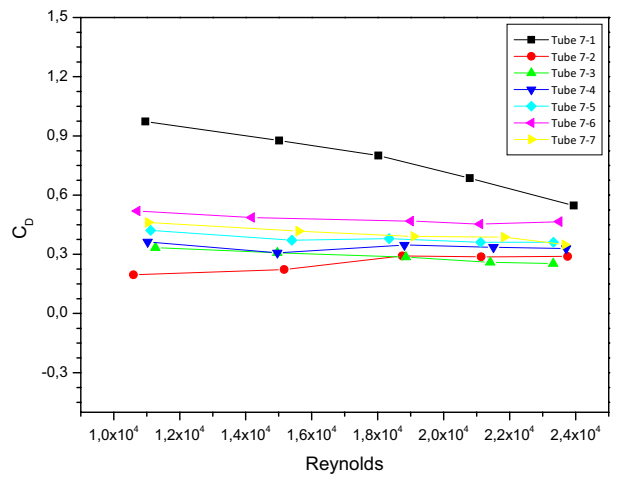

(g) Row 7

Fig. 8. Drag coefficient evolutions for each cylinder with a variation of Reynolds number. 
Table 2. Reduction of the pressure drop.

\begin{tabular}{|c|c|c|c|c|c|c|}
\hline$R e \times 10^{4}$ & & 1.13 & 1.33 & 1.6 & 1.87 & 2.13 \\
\hline \multirow{2}{*}{ Rate of the pressure drop reduction $[\%]$} & Case 1 & 22.9 & 29.4 & 9.1 & 14.6 & 5.8 \\
\hline & Case 2 & 33.8 & 36.5 & 25.7 & 22.1 & 11.7 \\
\hline
\end{tabular}

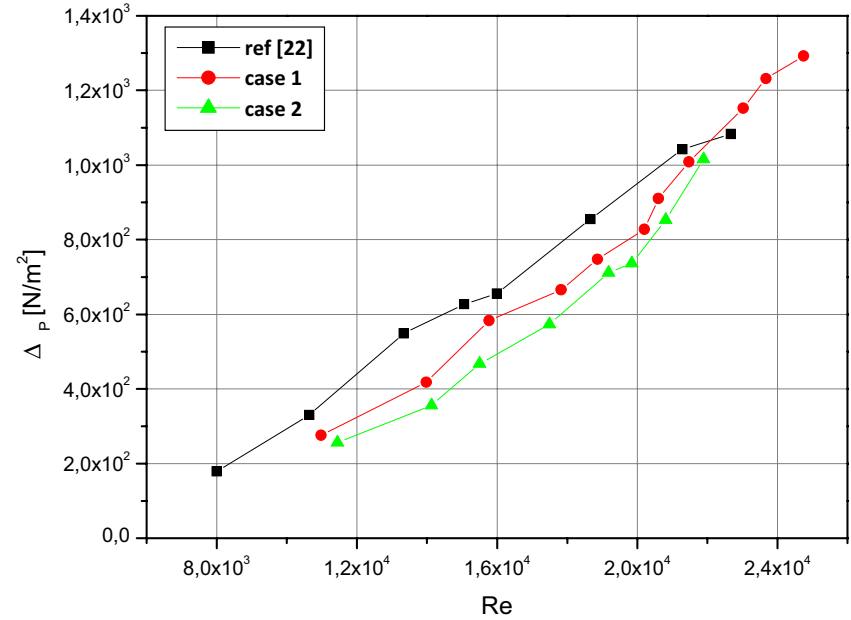

Fig. 9. Comparison of pressure drop for different Reynolds numbers.

From the above obtained results, two correlations with the best fitting represented by second order polynomial are suggested for the pressure drop across the tube bundle with the Reynolds number. For the first case the following equation is found:

$$
\Delta p_{\text {Case } 1}=123.82-(0.0142 \times R e)+\left(2.46 \times 10^{-6} \times(R e)^{2}\right)
$$

Otherwise the second case correlation is expressed as follows:

$\Delta p_{\text {Case } 2}=517.013-(0.071 \times R e)+\left(4.17 \times 10^{-6} \times(R e)^{2}\right)$

\section{Conclusion}

An experimental investigation of the turbulent flow around an in-line tube bundle for a pitch ratio of $P / D=1.44$ and a configuration of seven by seven tubes is performed. A passive flow control is used with longitudinal grooved cylinders. The results obtained are compared with the same smooth cylinder configuration and the following conclusions are drawn:

- Unsymmetrical pressure coefficients distributions at mid-span are obtained for all the rows except for row one. The shift of the stagnation point for the different rows results in flow direction changes in the tube bundle. Hence, the latter behaviour affects the aerodynamic forces on the different tubes.

- The force distributions along the tubes are nonuniform characterizing the $3 \mathrm{D}$ of the flow in the tube bundle.
- The complex interaction between the tubes in the bundle is exhibited by the drag interference distributions.

- The results found for the total drag allow some correlations to be obtained for the pressure drop function of Reynolds numbers.

- The use of the grooves gives a benefit effect for the total drag. The latter benefit of the grooves should be tested for the heat transfer and mechanical resistance.

\section{References}

[1] O. Pierson, Investigation of influence of tube arrangement on convection heat transfer and flow resistance in crossflow of gases in tube banks, ASME Trans. 59 (1937) 563572

[2] A. Zukauskas, R. Ulinskas, Heat Transfer in Tube Banks in Cross-Flow, Hemisphere, New York, 1988

[3] M. Arie, M. Kiya, M. Moriya, H. Mori, Pressure fluctuations on the surface of two cylinders in tandem arrangement, J. Fluids Eng. 105 (1983) 161-167

[4] Y.N. Chen, Fluctuating lift forces of the Karman vortex streets on single cylinders and tube bundles, part 3 - Lift forces in tube bundles, Trans. ASME, J. Engg. (1972). For Industry 94, 603-628; in Flow induced vibration of circular cylinder structures by S.S. Chen

[5] M.J. Pettigrew, P.L. Ko, A comprehensive approach to avoid vibration on fretting in shell and tube heat exchangers. Flow induced vibration of power plant components, PVP-41, ASME publications (1980), pp. 1-18; in Flow induced vibration of circular cylinder structures by S.S. Chen

[6] W. Wu, S. Huang, N. Barltrop, Current induced instability of two circular cylinders, Appl. Ocean Res. 24 (2002) 287-297

[7] I. Afgan, Industrial Applications of Large Eddy Simulation - Ph.D. thesis - University of Manchester, 2007

[8] S. Mittal, V. Kumar, Flow induced oscillations of two cylinders in tandem and staggered arrangements, J. Fluids Struct. 15 (2001) 717-736

[9] D. Wolfe, S. Zaida, Feedback control of vortex shedding from two tandem cylinders, J. Fluids Struct. 17 (2003) 579-592

[10] K. Lam, S.C. Lo, A visualization study of cross flow around four cylinders in a square configuration, J. Fluids Struct. 6 (1992) 109-131

[11] A.T. Sayers, Flow interference between four equispaced cylinders when subjected to a cross flow, Journal of Wind Engineering and Industrial Aerodynamics 31 (1988) 9-28

[12] A.T. Sayers, Vortex shedding from groups of three and four equispaced cylinders situated in cross-flow, Journal of Wind Engineering and Industrial Aerodynamics 34 (1990) 213-221 
[13] K. Lam, X. Fang, The effect of interference of four equispaced cylinders in cross flow on pressure and force coefficients, J. Fluids Struct. 9 (1995) 195-214

[14] K. Lam, S.C. Lo, A visualization study of cross-flow around four cylinders in a square configuration, J. Fluids Struct. 6 (1992) 109-131

[15] K. Lam, R.M.C. So, J.Y. Li, Flow around four cylinders in a square configuration using surface vorticity method, In Proceedings of the Second International Conference on Vortex Methods, Istanbul, Turkey, 2001

[16] K. Lam, J.Y. Li, K.T. Chan, R.M.C. So, Velocity map and flow pattern of flow around four cylinders in a square configuration at low Reynolds number and large spacing ratio using particle image velocimetry, In Proceedings of the Second International Conference on Vortex Methods, Istanbul, Turkey, 2001

[17] K. Lam, J.Y. Li, K.T. Chan, R.M.C. Son, The flow patterns of cross flow around four cylinders in an inline square configuration. In the Tenth International Symposium on Flow Visualization, Kyoto, Japan, 2002

[18] E.D. Grimison, Correlation and Utilisation of New Data on Flow Resistance and Heat Transfer for Crossflow of Gases over Tube Banks, Trans. ASME 59 (1937) 583-594

[19] A. Zukauskas, Heat Transfer from tubes in Crossflow, Adv. Heat Trans. 8 (1972) 93-160

[20] T. Yahiaoui, L. Adjlout, O. Imine, Experimental investigation of in-line tube bundles, Mechanika (2010) 37-43
[21] O. Ladjedel, T. Yahiaoui, L. Adjlout, O. Imine, Experimental and Numerical Studies of Drag Reduction on a Circular Cylinder, World Academy of Science, Eng. Technol. 77 (2011) 357-361

[22] T. Yahiaoui, Étude de l'influence des paramètres géométriques sur l'écoulement autour d'un faisceau de tubes, Thesis University of science and technology of Oran, Algeria, 2010

[23] P. Le Gal, I. Peschard, M.-P. Chauve, Y. Takeda, Collective behavior of wakes downstream a row of cylinders, Phys. Fluids 8 (1996) 2097

[24] S. Ishigai, E. Nishikawa, Experimental study of structure of gas flow in tube banks with tube axes normal to flow, Bull. J. Soc. Mech. (1975) Eng. 18528

[25] J.L. Auger, J. Coutanceau, Ecoulement transversal de l'air à travers une grille de tubes, Entropie 86 (1979) 13

[26] M. Hayashi, A. Sakurai, Y. Ohya, Wake interference of a row of normal flat plates arranged side by side in a uniform flow, J. Fluid Mech. 164 (1986) 1

[27] M.M. Zdravkovich, D.L. Pridden, Interference between two circular cylinders; series of unexpected discontinuities, J. Ind. Aerodyn 2 (1977) 255-270

[28] M.A. Mehrabian, Heat transfer and pressure drop characteristics of cross flow of air over a circular tube in isolation and/or in a tube bank, The Arabian J. Sci. Eng. $32(2007)$ 